\title{
Mesenchymal stem cells: a double-edged sword in regulating immune responses
}

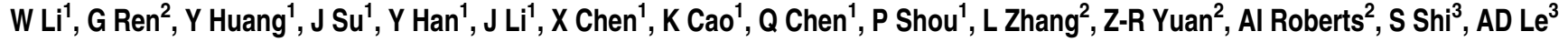 \\ and $Y$ Shi $^{, 1,2}$
}

Mesenchymal stem cells (MSCs) have been employed successfully to treat various immune disorders in animal models and clinical settings. Our previous studies have shown that MSCs can become highly immunosuppressive upon stimulation by inflammatory cytokines, an effect exerted through the concerted action of chemokines and nitric oxide (NO). Here, we show that MSCs can also enhance immune responses. This immune-promoting effect occurred when proinflammatory cytokines were inadequate to elicit sufficient NO production. When inducible nitric oxide synthase (iNOS) production was inhibited or genetically ablated, MSCs strongly enhance T-cell proliferation in vitro and the delayed-type hypersensitivity response in vivo. Furthermore, iNOS $^{-l-}$ MSCs significantly inhibited melanoma growth. It is likely that in the absence of NO, chemokines act to promote immune responses. Indeed, in $\mathrm{CCR}^{-1-} \mathrm{CXCR3}^{-1-}$ mice, the immune-promoting effect of $i \mathrm{NOS}^{-1-} \mathrm{MSC}$ is greatly diminished. Thus, NO acts as a switch in MSC-mediated immunomodulation. More importantly, the dual effect on immune reactions was also observed in human MSCs, in which indoleamine 2,3-dioxygenase (IDO) acts as a switch. This study provides novel information about the pathophysiological roles of MSCs.

Cell Death and Differentiation (2012) 19, 1505-1513; doi:10.1038/cdd.2012.26; published online 16 March 2012

Mesenchymal stem cells (MSCs) exist in almost all tissues to generate replacements for cells that are lost through normal aging, injury or disease. These cells have been successfully isolated from various tissues such as bone marrow, muscle, fat, brain, heart, skin and Wharton's jelly of umbilical cord. These cells provoke few ethical issues and do not form teratomas. They also have immunomodulatory effects. Thus, MSCs hold great promise for treating various diseases. In fact, in vitro expanded MSCs have already been applied in preclinical and clinical studies to promote successful umbilical cord blood stem cell transplantation, ${ }^{1}$ and to treat diseases such as GvHD, ${ }^{2,3}$ type I diabetes, ${ }^{4}$ multiple sclerosis, ${ }^{5,6}$ liver cirrhosis, ${ }^{7}$ systemic lupus erythematosus ${ }^{8}$ and Crohn's disease..$^{9,10}$ These studies collectively demonstrate the great clinical potential of MSCs. According to the clinical trial registry at the National Institute of Health, over one hundred clinical trials with these unique cells are currently being conducted (http://clinicaltrials.gov/ct2/home).

The most important clinically relevant characteristics of MSCs are their readiness to grow in culture and their immunosuppressive capacity. MSCs can be highly immunosuppressive, and have been found to suppress T-cell proliferation and cytokine production. ${ }^{11,12}$ We recently reported that MSCs prevent the rejection of allogeneic skin grafts $^{13}$ and also ameliorate GvHD in mice. ${ }^{14}$ Other studies have variously demonstrated that MSC-mediated immunosuppression involves IL-10, ${ }^{15}$ TGF- $\beta,{ }^{16} \mathrm{NO},{ }^{12}$ IDO (indoleamine 2,3-dioxygenase), ${ }^{17}$ tumor necrosis factor (TNF) -stimulated gene-6 (TSG6) ${ }^{18}$ and prostaglandin (PG) E2. ${ }^{19}$ Our systematic analysis revealed that the immunosuppressive effect of MSCs depends on interferon-gamma (IFN $\gamma$ ) in the co-presence of another cytokine: TNF $\alpha, \mathrm{IL}-1 \alpha$ or IL- $1 \beta$. Such cytokine pairs provoke MSCs to express inducible nitric oxide synthase (iNOS), produce NO, and secrete large amounts of chemokines. These chemokines, in turn, complement the activity of $\mathrm{NO}$ in co-cultures, by driving $\mathrm{T}$ cell to migrate into the proximity with MSCs, where the high levels of NO suppress the T cells. Like mouse MSCs, human and nonhuman primate MSCs have a similar spectrum of chemokine production, but they utilize IDO instead of NO to suppress T-cell function. ${ }^{20}$

Interestingly, the immunosuppressive capacity of MSCs is not always achieved. Some researchers have claimed that MSCs were unable to protect mice from GvHD although they did suppress lymphocyte proliferation in vitro to some degree. ${ }^{21}$ Accelerated graft rejection after MSCs administration has also been observed. In allogeneic bone marrow transplantation in sublethally irradiated mice, co-transfusion

\footnotetext{
${ }^{1}$ Key Laboratory of Stem Cell Biology, Institute of Health Sciences, Shanghai Institutes for Biological Sciences, Chinese Academy of Sciences/Shanghai Jiao Tong University School of Medicine, 225 South Chongqing Road, Shanghai 200025, China; ${ }^{2}$ Child Health Institute of New Jersey, University of Medicine and Dentistry of New Jersey-Robert Wood Johnson Medical School, 89 French Street, New Brunswick, NJ 08901, USA and ${ }^{3}$ Center for Craniofacial Molecular Biology, University of Southern California School of Dentistry, 2250 Alcazar Street, CSA 103, Los Angeles, CA 90033, USA

${ }^{*}$ Corresponding author: Y Shi, Key Laboratory of Stem Cell Biology, Institute of Health Sciences, Shanghai Institutes for Biological Sciences, 225 South Chongqing Road, Shanghai 200025, China. Tel: + 8621 63848329; Fax: + 8621 63846467; E-mail: yufangshi@sibs.ac.cn

Keywords: mesenchymal stem cells; chemokine; immunomodulation; tissue repair

Abbreviations: MSCs, mesenchymal stem cells; NO, nitric oxide; iNOS, inducible nitric oxide synthase; IDO, indoleamine 2,3-dioxygenase; DTH, delayed-type hypersensitivity; IFN $\gamma$, interferon-gamma; TNF $\alpha$, tumor necrosis factor-alpha; OVA, ovalbumin; CFA, complete Freud's adjuvant; SNAP, S-nitroso-N-acetyl-DLpenicillamine; CFSE, carboxy fluorescein diacetate succinimidyl ester; L-NMMA, NG-monomethyl-L-arginine acetate salt; SMT, S-methyl-isothiourea; 1400W, N-(3(aminomethyl)benzyl) acetamidine dihydrochloride; PBMCs, peripheral blood mononuclear cells

Received 14.11.11; revised 13.2.12; accepted 13.2.12; Edited by G Melino; published online 16.3.12
} 
of MSCs has been reported to result in significantly decreased engraftment of donor cells. ${ }^{22}$ Similarly, another study showed that MSCs did not prevent graft rejection in vivo. Surprisingly, combined administration of both MSCs and cyclosporine A, a universally used immunosuppressant, actually accelerated graft rejection. ${ }^{23}$ Interestingly, it has been shown that MSCs do not enhance drug-induced; instead, MSCs restored the normal proliferation of peripheral blood mononuclear cells (PBMCs). ${ }^{24}$ Given these apparently contradictory findings, the immunosuppressive capability of MSCs required further exploration.

In clinical trials, MSCs have been reported to be effective in the treatment of steroid-refractory GvHD. ${ }^{2,25}$ Notably, some patients showed no improvement or even worsened clinical condition after MSCs therapy, but these results were not emphasized in the reports. Thus, MSC-based cell therapy may actually be harmful to a subset of patients. ${ }^{26}$ On the basis of our previous understanding of a concerted action of chemokines and NO in MSC-mediated immunosuppression, we hypothesize that iNOS acts as a switch in determining whether MSCs will suppress or enhance the immune response.

In this study, we employed iNOS inhibitors, and iNOSdeficient MSCs, and found that MSCs can indeed enhance immune responses under certain conditions. With low densities of T cells or with suboptimal activation, MSCs were able to promote immune responses due to their production of chemokines but very low levels of NO. Notably, when iNOS was inhibited or genetically ablated in the MSCs, lymphocyte proliferation in vitro was dramatically upregulated by MSCs. Such immune-enhancement by MSCs in the absence of iNOS activity could also be demonstrated in vivo using the delayedtype hypersensitivity (DTH) assay, and this effect was largely reverted in $\mathrm{CCR}^{-1-}{ }^{-} \mathrm{CXCR}^{-1-}$ mice. Therefore, chemokines secreted by inflammatory cytokine-stimulated MSCs that are responsible for their immune enhancement effect when these cells poorly express iNOS. Similarly, in human MSCs, such a balance is determined by chemokines and IDO. Thus, immune enhancement by MSCs is generated by chemokine-mediated immune cell aggregation in the absence of immunosuppressive effector molecules. We believe that our findings that MSCs can enhance immune responses provides critical information for a better understanding of the role of MSCs in various pathological conditions and for developing better protocols for the clinical application of MSCs.

\section{Results}

MSC-mediated immunosuppression requires sufficient proinflammatory cytokines. To examine the interplay between MSCs and immune responses, we derived MSCs from mouse bone marrow according to established protocols. ${ }^{27,28}$ All cells used were capable of differentiating into adipocytes and osteoblasts when cultured under appropriate conditions and possess the following pheno-

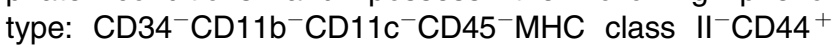
Sca- $1^{+} \mathrm{MHC}$ class $\mathrm{I}^{\text {low }},{ }^{13,14}$ confirming the stem cell properties of our MSCs.
Our previous studies have shown that proinflammatory cytokines produced by activated lymphocytes can stimulate mouse MSCs to produce both chemokines and NO, which then act in concert to inhibit the proliferation of T lymphocytes: the chemokines attract lymphocytes into close proximity with the MSCs, where high concentrations of NO in the microenvironment suppress T-cell function. ${ }^{14}$ In our studies, MSCs from passage 5 through passage 15 were used, and their ability to produce NO in the presence of TNF $\alpha$ and IFN $\gamma$ did not change with passaging (Supplementary Figure S1A). To assay the efficacy of inflammatory cytokines to induce immunosuppression by MSCs, we added graded concentrations of IFN $\gamma$ and TNF $\alpha$ to MSCs co-cultured with T-cell blasts, which produce very little cytokine unless re-stimulated, and examined the effect on their proliferation. We found that MSCs were highly responsive to low levels of cytokines: significant induction of immunosuppression was observed when as little as $0.4 \mathrm{ng} / \mathrm{ml}$ of each cytokine was added, whereas there was no effect without cytokine addition (Figure 1a). This inhibition on $\mathrm{T}$ cells was dependent on NO, as the iNOS inhibitor NGmonomethyl-L-arginine acetate salt (L-NMMA) completely restored $\mathrm{T}$-cell blast proliferation in the co-culture regardless of the concentration of IFN $\gamma$ and TNF $\alpha$ added (Figure 1b). Thus, the optimal immunosuppression capacity in MSCs depends on $\mathrm{NO}$ and does not require high amount of IFN $\gamma$ and $\mathrm{TNF} \alpha$.

MSCs can stimulate splenocyte proliferation when NO production is low. Under physiological conditions, it is unlikely that MSCs in situ are always immunosuppressive. Rather, the immunomodulatory effect of MSCs probably varies depending on the immune status of the microenvironment. We hypothesize that, under conditions in which activated $\mathrm{T}$ cells produce insufficient levels of IFN $\gamma$ and $\mathrm{TNF} \alpha$ to induce immunosuppression, MSCs can instead enhance T-cell proliferation. To test this hypothesis, MSCs were co-cultured with freshly isolated splenocytes at various ratios in the presence of anti-CD3 to stimulate the TCR and the effect on proliferation was determined. It should be noted that, in contrast to T-cell blasts, splenocytes activated with anti-CD3 secrete large amounts of inflammatory cytokines including IFN $\gamma$ and TNF $\alpha$. As shown in Figure 2a, in absence of MSCs the proliferation of anti-CD3-stimulated splenocytes exhibited typical cell-density dependence. On the other hand, in the presence of MSCs, the proliferation of T cells was inhibited at the higher splenocytes densities, whereas it was actually enhanced at lower densities, indicating that MSCs can be either immunosuppressive or immune enhancing, depending on the concentration of T cells.

We next examined the effect of MSCs on T-cell activation using different amounts of anti-CD3, as we predicted that MSCs are capable of enhancing the proliferation of suboptimally activated $\mathrm{T}$ cells while inhibiting optimallyactivated T cells. Therefore, freshly isolated splenocytes were activated with graded amounts of anti-CD3, in the presence or absence of MSCs. As shown in Figure 2c, in the presence of low concentrations of anti-CD3, the presence of MSCs significantly enhanced T-cell proliferation. In contrast, at higher concentrations of anti-CD3, T-cell proliferation was inhibited by MSCs. 
a IFNy (ng/ml)

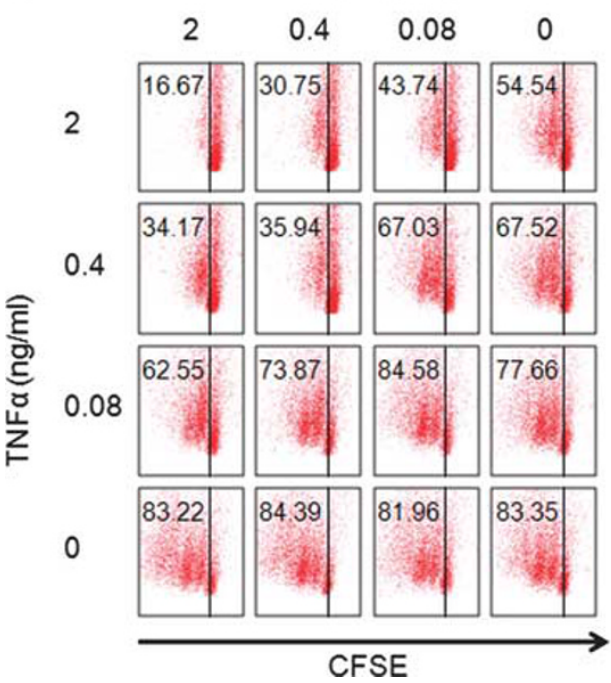

b IFNy $(\mathrm{ng} / \mathrm{ml})$

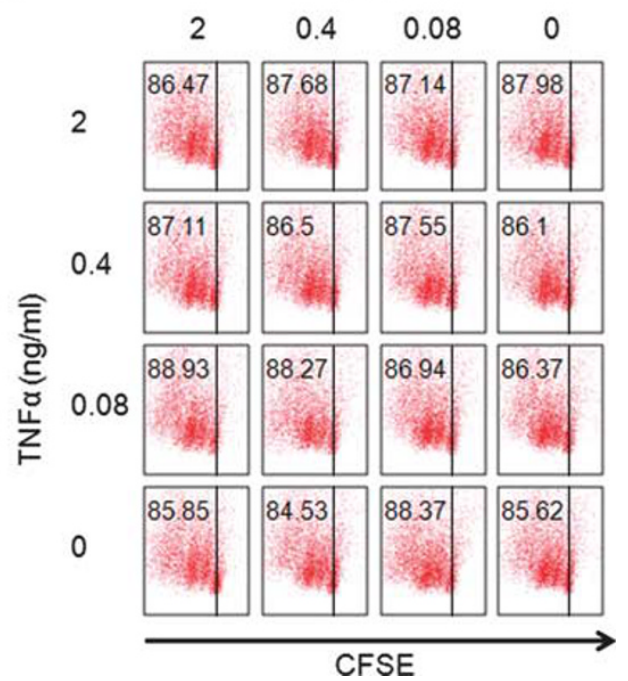

Figure 1 Proinflammatory cytokines induce immunosuppression by MSCs in a cytokine dose-dependent and NO-dependent manner. T-cell blasts stained with CFSE were co-cultured with MSCs (10000 cells/well in 96-well plate) at a 1: 10 ratio (MSCs:T cells) in the presence of various concentrations of IFN $\gamma$ and TNF $\alpha$ in IL-2 (200 U/mI)containing medium (a). L-NMMA (1 mM) was added at the beginning of co-culture (b). $48 \mathrm{~h}$ later, all the cells were subjected to flow cytometry for T-cell proliferation detection as indicated by the reduction in CFSE intensity. The percentage of proliferated cells was indicated (\%). Data are representative of four independent experiments
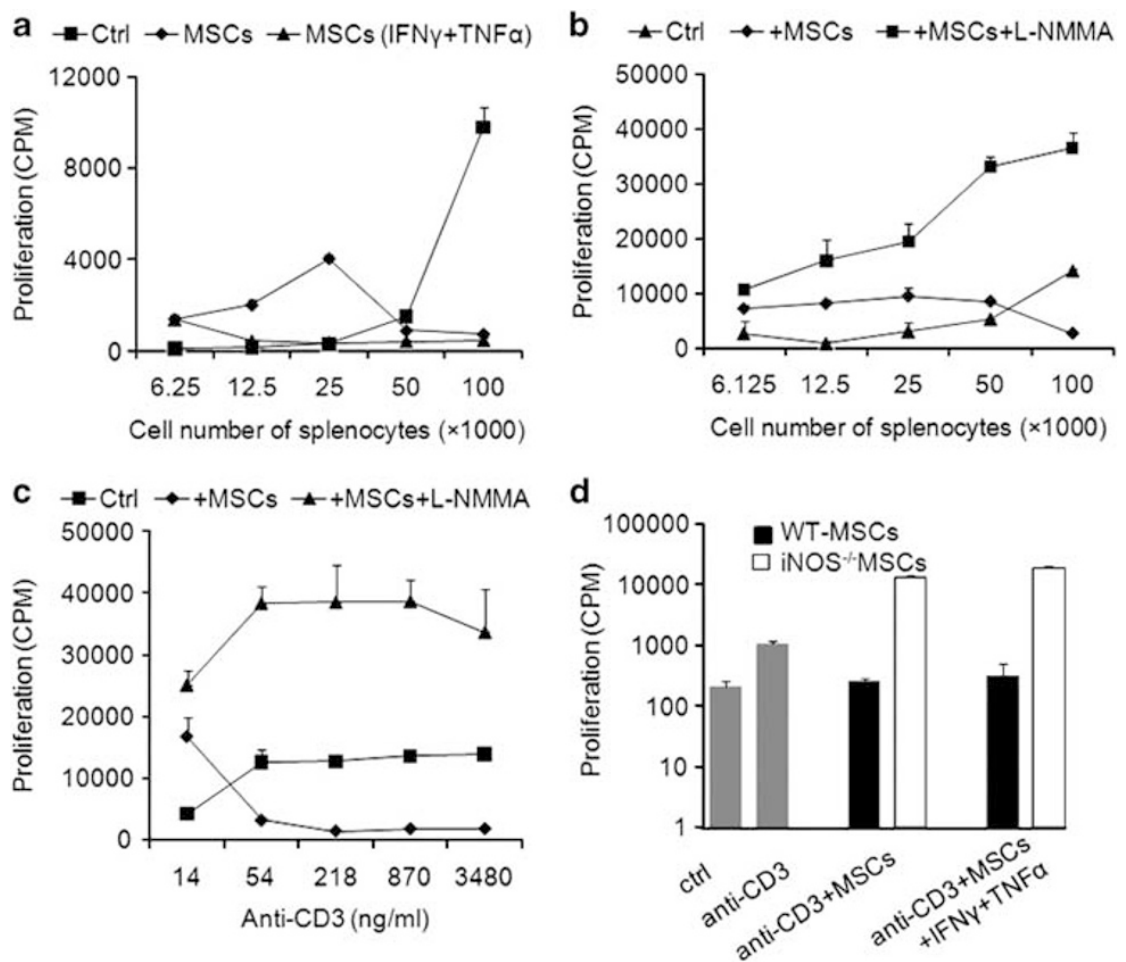

Figure 2 MSCs promote T-cell activation when NO is insufficient. (a) Different number of splenocytes were activated with anti-CD3 (0.87 $\mu \mathrm{g} / \mathrm{ml})$ and $\mathrm{co-cultured} \mathrm{with}$ MSCs (5000 cells/well in 96-well plate) with and without IFN $\gamma+$ TNF $\alpha(2 \mathrm{ng} / \mathrm{ml}$ each) pretreatment at the indicated ratios, in the absence or presence of iNOS inhibitor, LNMMA ( $1 \mathrm{mM})$. (b) T-cell proliferation was measured $48 \mathrm{~h}$ later. (c) Splenocytes $\left(1 \times 10^{5}\right.$ per well in 96 -well plate) were activated with different concentrations of anti-CD3 cocultured with MSCs (1:10, MSC: splenocytes) with and without L-NMMA (1 mM). (d) Wild-type MSCs and iNOS ${ }^{-1-}$ MSCs (5000 cells/well in 96-well plate) were co-cultured with splenocytes at a 1:10 ratio with or without anti-CD3 $(0.87 \mu \mathrm{g} / \mathrm{ml})$, in the presence or absence of IFN $\gamma$ plus TNF $\alpha(2 \mathrm{ng} / \mathrm{ml}$ each). Data shown are means $+\mathrm{S}$.E. of a representative of three independent experiments

We reasoned that the immune-enhancing effect of MSCs is likely due to insufficient levels of inflammatory cytokines. Therefore, we pretreated MSCs with an optimal amount of
IFN $\gamma$ and TNF $\alpha$ and then co-cultured them with splenocytes at graded densities. We found that under these conditions, T-cell proliferation was strikingly inhibited even at low T-cell 
densities (Figure 2a). Thus, the concentration of proinflammatory cytokines determines whether MSCs will become immunosuppressive or immune-enhancing.

We have previously reported that NO has a central role in MSC-mediated immunosuppression. ${ }^{14} \mathrm{NO}$ production by MSCs is tightly regulated by proinflammatory cytokines and iNOS is its sole source. Two other NOS isoforms were undetectable in MSCs, with or without proinflammatory cytokine stimulation. Furthermore, mouse MSCs deficient in iNOS did not produce NO even when IFN $\gamma$ and TNF $\alpha$ were present (Supplementary Figure S2). Nitrate production by MSCs increased with increasing concentrations of supplemented cytokines (Supplementary Figure S1B). To test our hypothesis that the NO mediates immunosuppression, we utilized L-NMMA, an iNOS inhibitor, to block NO production and examined the immunomodulatory effect of MSCs. Indeed, we found that when NO production is inhibited, MSCs boosted splenocyte proliferation at all levels of anti-CD3 activation (Figure $2 b, c)$ ). Also, we found that two other non-competitive iNOS inhibitors, S-methyl-isothiourea (SMT) sulfate and $\mathrm{N}$-(3-(aminomethyl)benzyl) acetamidine dihydrochloride (1400w), had similar effects on NO production and enhancement of splenocyte proliferation (Supplementary Figure S3). Thus, blockade of iNOS activity could enable MSCs to become immune enhancing. To further verify the role of NO in modulating the proliferation of activated T cells, we employed iNOS ${ }^{-1}$ MSCs. When co-cultured with anti-CD3activated splenocytes, iNOS ${ }^{-1-} \mathrm{MSCs}$ were not only incapable of suppressing lymphocyte proliferation, but also caused dramatically increased proliferation (Figure 2d). Furthermore, addition of exogenous IFN $\gamma$ and TNF $\alpha$ also enhanced the immune response in the presence of iNOS-deficient MSCs (Figure 2d). Thus, without NO production, MSCs enhance rather than inhibit lymphocyte proliferation. Taken together, these results indicate that MSCs can either suppress or promote immune responses; depending on the levels of NO they produce.

MSCs aggravate DTH in the absence of NO. As we showed above, MSCs are able to promote immune responses when NO production is blocked. On the basis of this observation, we predicted that $\mathrm{iNOS}^{-1-}$ MSCs would aggravate an immune response. To test this hypothesis in vivo, we used a mouse model of DTH response to examine the effect of administration of iNOS $^{-1-}$ MSCs. Ovalbumin (OVA) in complete Freund's adjuvant was administrated at the tail base. On day 7 the animals were re-challenged by heat-denatured OVA co-administered with wild-type MSCs or iNOS ${ }^{-1-}$ MSCs by injection into one hind footpad. The resultant DTH responses were measured by footpad thickness increment and by histological examination using H\&E staining. As expected, wild-type MSCs significantly reduced the DTH response. In contrast, mice injected with $\mathrm{iNOS}^{-1-}$ MSCs exhibited a significantly enhanced DTH response in comparison with mice treated with OVA alone (Supplementary Figure S4A). ${ }^{14}$ Similarly, when iNOS inhibitor L-NMMA was administered together with wild-type MSCs, the DTH response was also boosted (Supplementary Figure S5). Moreover, on histological sections, alleviation of inflammation response was also observed in mice co-injected with wild-type MSCs, while there was significantly more leukocyte infiltration in mice treated with iNOS $^{-1-}$ MSCs (Supplementary Figure S4B). ${ }^{14}$ Meanwhile, an increase in lymphocyte infiltration was also seen in mice treated with MSCs and L-NMMA (data not shown). Therefore, in this in vivo model, MSCs were again found to have a dual role, either immunosuppressive or immune enhancing, depending on the status of their NO production.

Chemotaxis has a critical role in MSC-mediated immune enhancement. We recently demonstrated that during inflammatory cytokine-activated MSC-mediated immunosuppression, chemokines attract immune cells into close proximity with MSCs, where locally high concentrations of NO can suppress lymphocyte function. To test the ability of $\mathrm{NO}$ to suppress activation-induced T-cell proliferation, we added the NO donor S-nitroso-acetylpenicillarnine (SNAP) to anti-CD3-activated splenocyte cultures. As a control for the effect of SNAP, we used NO promoted Fasmediated apoptosis and found that $28 \mu \mathrm{M}$ was effective (Supplementary Figure S6A, B), whereas inhibition of T-cell proliferation required $>60 \mu \mathrm{M}$ (Supplementary Figure S6C, D). Therefore, T-cell proliferation was suppressed only when SNAP was added at high concentration. Interestingly, when $0.4 \mathrm{ng} / \mathrm{ml}$ each of IFN $\gamma$ and TNF $\alpha$ were added to the coculture of MSCs and T-cell blasts, there was significant inhibition of T-cell proliferation (Figure 1) but only a moderate amount of total NO was produced (Supplementary Figure S1B). Therefore, in cytokine-activated MSC cultures, biologically active NO is not uniformly distributed. It is likely that $\mathrm{T}$ cells need to be attracted into close proximity with MSCs, where higher concentrations of biologically-active $\mathrm{NO}$ exist. In the absence of $\mathrm{NO}$, as occurs with iNOS ${ }^{-1-}$ MSCs, it is likely that the large amounts of MSC-produced chemokines mediate the immune-enhancing effect of these cells by recruiting lymphocytes into their vicinity. In our DTH assay, for example, co-administration of $\mathrm{iNOS}^{-1-} \mathrm{MSCs}$ upon re-challenge with OVA resulted in a much greater incidence of infiltrating leukocytes in the footpad.

To examine the role of chemokines, we firstly examined if their expression was affected when iNOS activity was blocked. As shown in Figure 3, neither iNOS inhibitors nor genetic ablation of iNOS had any influence on chemokine expression by MSCs co-stimulated with IFN $\gamma$ and TNF $\alpha$. In addition, cytokine-stimulated iNOS ${ }^{-1-}$ MSCs produced typical levels of other factors such as IL-6, implying that NO does not affect the responsiveness of MSCs to proinflammatory cytokines in general (Supplementary Figure S7). We have reported that the chemokines most potently upregulated by inflammatory cytokines in MSCs are the ligands for two prominent chemokine receptors on T cells, CCR5 and CXCR3. ${ }^{14}$ To validate the roles of chemokines in MSCmediated immune-enhancement, we compared the immuneenhancing effect of MSCs on wild-type and $\mathrm{CXCR}^{-1-}$ $\mathrm{CCR}^{-1-}$ splenocytes. Both types of splenocytes were activated with anti-CD3 in the presence of $\mathrm{iNOS}^{-1-}$ MSCs at 2500 per well in 96-well plates. Under these conditions, iNOS $^{-1-}$ MSCs strongly promoted wild-type splenocyte proliferation, whereas such effect on $\mathrm{CXCR}^{-1-} \mathrm{CCR} 5^{-1-}$ splenocyte was dramatically reduced (Figure 4a), demon- 
WT-MSCS $\square$ iNOS $\%$ MSCS

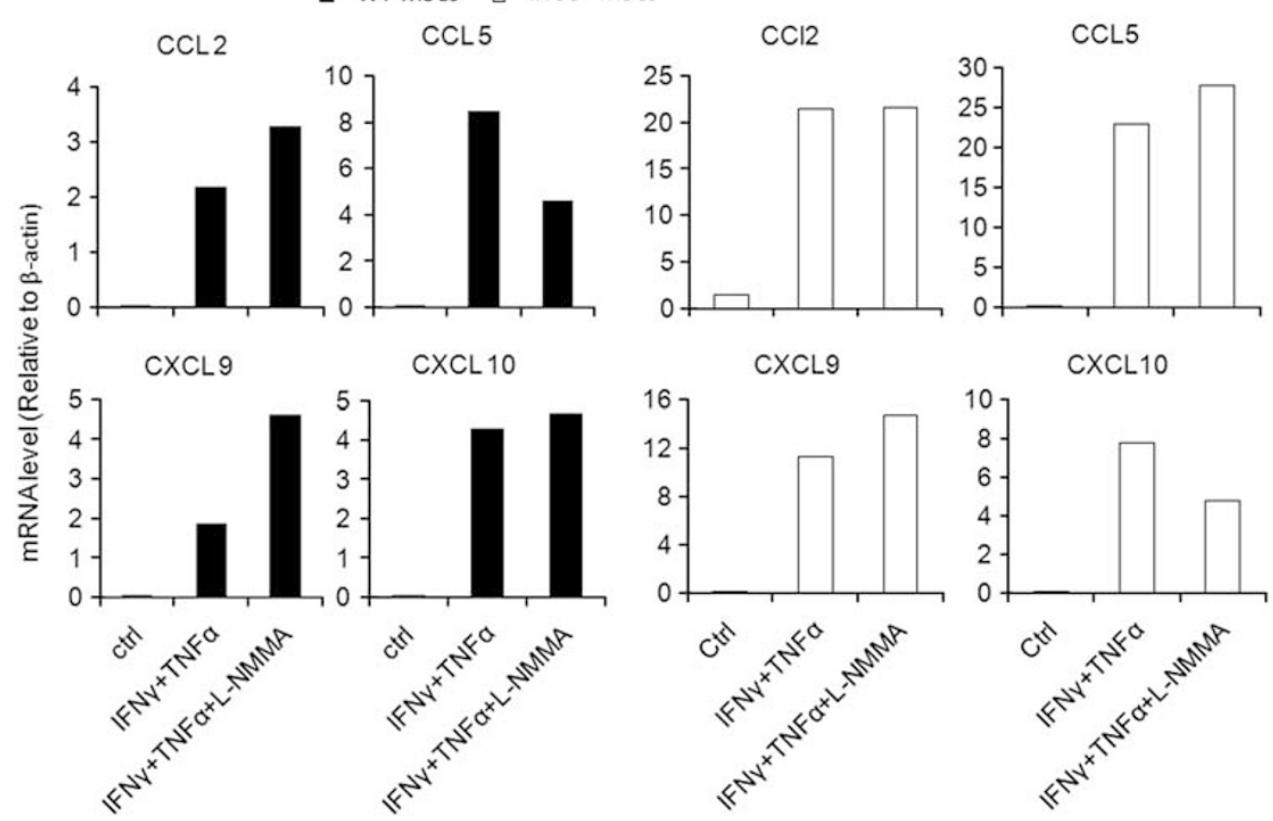

Figure 3 Proinflammatory cytokines induce high level of chemokine expression in MSCs regardless of NO production. Wild-type MSCs and iNOS ${ }^{-1-}$ MSCs $\left(5 \times 10^{5}\right.$ cells/ well in 6-well plates) were stimulated with IFN $\gamma$ and TNF $\alpha(2 \mathrm{ng} / \mathrm{ml}$ each) for $12 \mathrm{~h}$, and L-NMMA was added in the groups indicated. The expression of chemokines was assayed by real-time PCR. Data are representative of three independent experiments
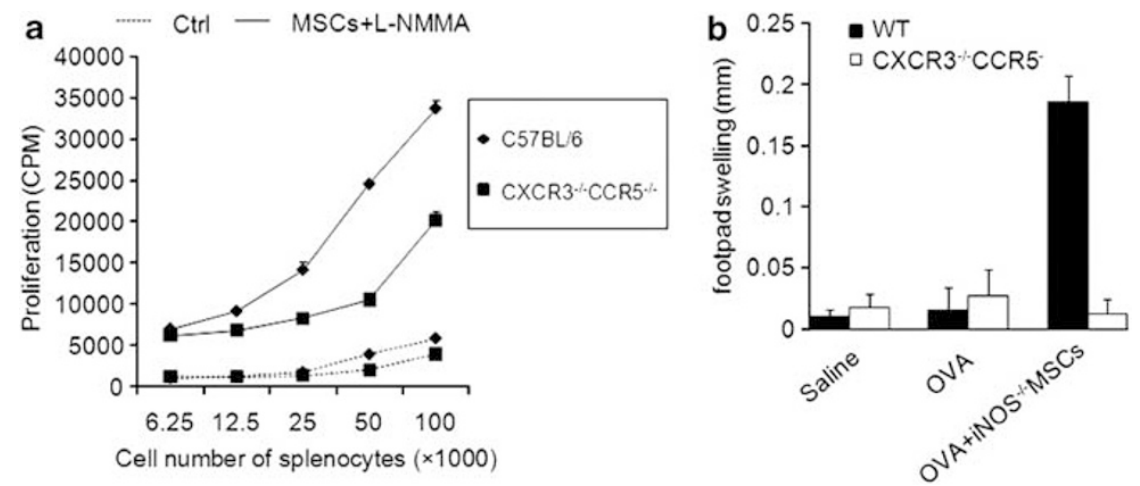

Figure 4 MSCs could not promote DTH response in $\mathrm{CCR}^{-1-} \mathrm{CXCR}^{-1-}$ mice. (a) Freshly isolated splenocytes from C57BL/6 or $\mathrm{CXCR} 3^{-1-} \mathrm{CCR} 5^{-1-}$ mice (on the C57BL/6 background) at different cell density were co-cultured with wild-type MSCs (2500 cells/well in 96-well plates) in the presence of L-NMMA(1 mM), and anti-CD3 $(0.87 \mu \mathrm{g} / \mathrm{ml})$ was added at the beginning of co-culture. (b) C57BL/6 $(n=5)$ and CXCR3 ${ }^{-1-}$ CCR $^{-1-}(n=5)$ mice were immunized with OVA $(10 \mu \mathrm{g}$ in $50 \mu \mathrm{l} \mathrm{saline)} \mathrm{emulsified}$ with $50 \mu \mathrm{l}$ complete Freund's adjuvant. On day 7, mice were challenged in the footpad with $20 \mu \mathrm{g}$ OVA administered with or without wild-type or iNOS ${ }^{-1-}{\text { MSCs }\left(2.5 \times 10^{5}\right.}^{5}$ cells). Footpad thickness increment was determined after $24 \mathrm{~h}$ as a measurement of DTH response. Data shown are means $+\mathrm{S}$.E. of a representative of three independent experiments

strating an important role for chemotaxis in MSC-mediated immune-enhancement.

To examine the role of chemokines in MSC-mediated immune enhancement in vivo, we employed the DTH model and compared the responses in wild-type and $\mathrm{CXCR}^{-/-}$ $\mathrm{CCR}^{-1-}$ mice. We utilized a suboptimal dose of OVA $(20 \mu \mathrm{g})$ to challenge the animals, so that it would be possible to detect immune enhancement by iNOS $^{-/-}$MSCs. At this low level of antigen, although there was little swelling in the wild-type control mice, significant swelling did occur in the presence of iNOS $^{-1-}$ MSCs. Interestingly, such immune enhancement by iNOS $^{-1-}$ MSCs was not detected in $\mathrm{CXCR}^{-1-} \mathrm{CCR}^{-1-}$ mice (Figure 4b). This result suggests that chemokines are crucial factors in immune enhancement mediated by iNOS $^{-1-}$ MSCs.
iNOS $^{-l-}$ MSCs suppress tumor growth. The immune response is critical in controlling tumor growth. A tumor can be considered to be a wound that never heals, and thus tumor sites always attract MSCs. These stem cells could therefore have a critical role in regulating tumor growth. As we have shown that iNOS-deficient MSCs can enhance immune responses in vitro and in vivo, we asked if iNOS ${ }^{-1-}$ MSCs might be able to promote the anti-tumor immunity in vivo. We tested this possibility in a mouse model of melanoma. B16-F0 melanoma cells were co-administered with wild-type MSCs or $\mathrm{iNOS}^{-1-}$ MSCs, and 14 days later, the resultant tumors were weighed. Infusion of $\mathrm{iNOS}^{-1-}$ MSCs, but not wild-type MSCs, was found to significantly reduce tumor growth (Figure 5a). Similarly, an anti-tumor 
effect by wild-type MSCs was also observed when the iNOS inhibitor L-NMMA was co-administered (Supplementary Figure S8). More importantly, neither WT nor iNOS ${ }^{-1-}$ MSCs could affect melanoma growth in immunodeficient mice, implying the involvement of the adaptive immunity in the iNOS $^{-1-}$ MSC-mediated anti-tumor effect. This was further evidenced by histological analysis of $\mathrm{CD}^{+}$cell infiltration in tumor sections. At tumor sites, iNOS ${ }^{-1-} \mathrm{MSCs}$ were able to recruit significantly more $\mathrm{CD}^{+}$cells, compared with WT MSCs (Figure 5b). Therefore, modulation of iNOS activity could provide a novel strategy for tumor immunotherapy.

IDO knockdown makes human MSCs immune-enhancing. We have shown that mouse MSCs can promote immune responses when exposed to low dose of proinflammatory cytokines or in the absence of iNOS activity. Next, we examined whether the same holds true for human MSCs. As reported previously, we found that, like mouse MSCs, human MSCs also produce abundant chemokines such as CXCL9, CXCL10, CXCL11 upon stimulation with proinflammatory cytokines (Supplementary Figure S9). Unlike mouse MSCs, however, human MSCs utilize IDO to suppress immune responses, ${ }^{20}$ and we found no detectable levels of iNOS or the other two NOS isoforms (Supplementary Figure S9). Therefore, in MSC-mediated immunosuppression, there is a very obvious species variation. To this end, we knocked down IDO using

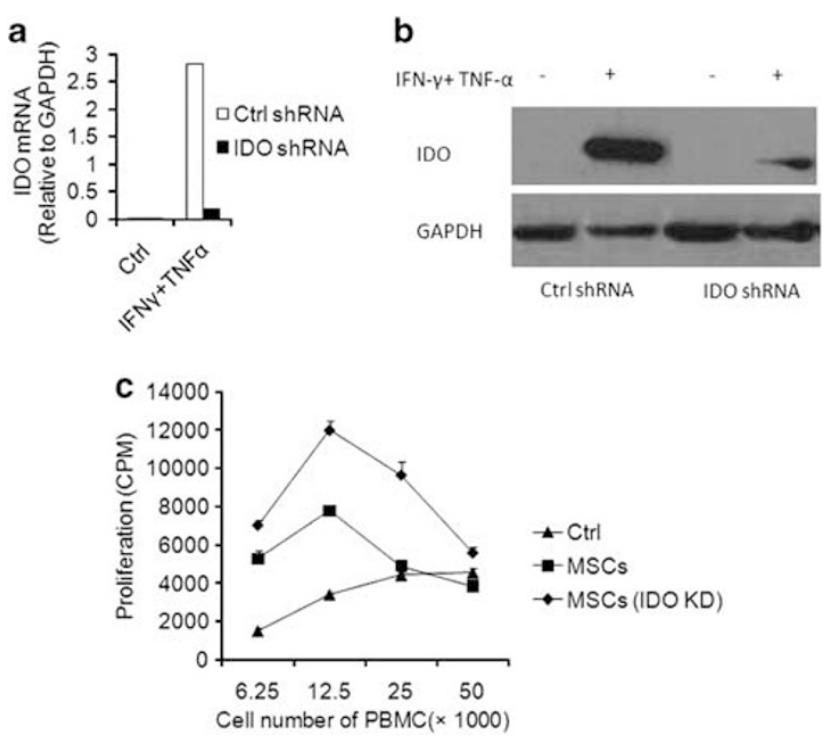

Figure 6 Human MSCs with IDO knockdown are immune enhancing. Human umbilical cord-derived MSCs were infected by IDO shRNA lentiviral particles or control shRNA lentiviral particles for $24 \mathrm{~h}$. Puromycin at $3 \mathrm{mg} / \mathrm{ml}$ was then added to the culture after removal of lentiviral particles. After 3 days, the cells were collected for the detection of IDO at both mRNA level (a) and protein level (b). Both MSCs (5000 cells/well in 96-well plate) were co-cultured with different number of PBMCs isolated from health donors in the presence of anti-CD3 at $0.5 \mu \mathrm{g} / \mathrm{ml}$. After $72 \mathrm{~h}$ tritium thymidine at $0.5 \mu \mathrm{ci} /$ well was added and incubated for $6-8 \mathrm{~h}$. Tritium thymidine incorporation was detected by scintillation counting (c)

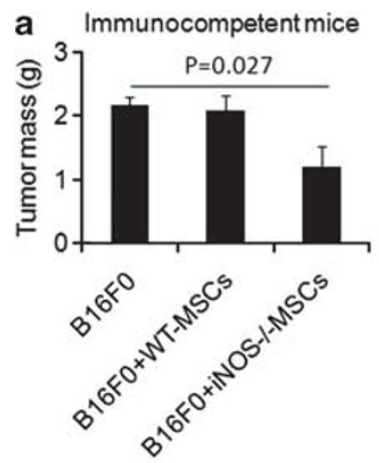

b
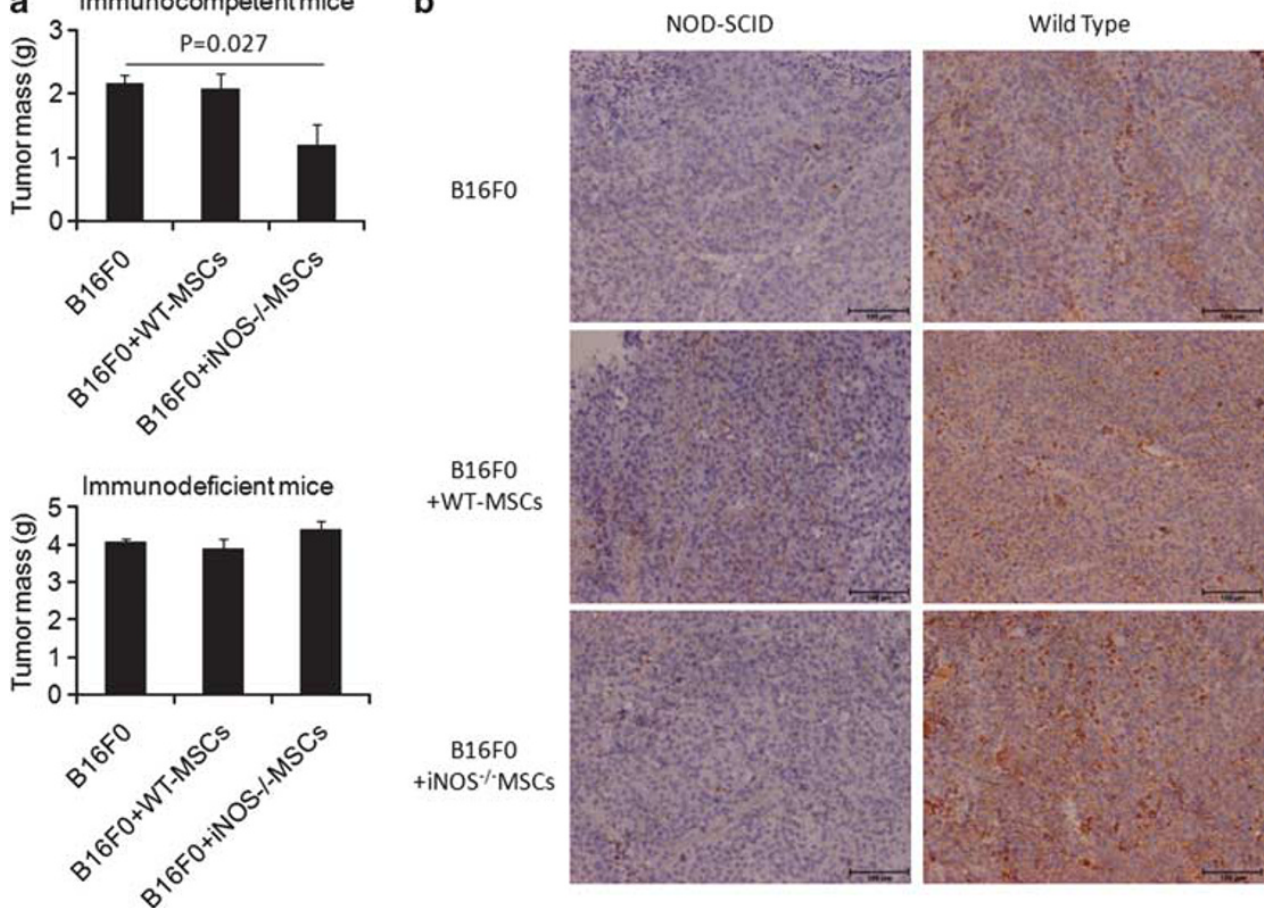

Figure 5 iNOS-deficient MSCs suppress melanoma growth. (a) At day 0, B16-F0 cells $\left(0.5 \times 10^{6}\right.$ cells) were administrated intramuscularly in the outside of left thigh of

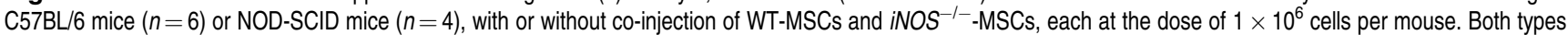
of MSCs were further administrated at day 3, 6 and 9 at the same sites with the original injection, with PBS as a control. After 14 days, the mice were killed and the tumors were weighed. (b) Tumor sections were prepared for histological analysis of $\mathrm{CD} 3^{+}$cells. Data shown are means $+\mathrm{S}$.E. of a representative of three independent experiments 
shRNA in human MSCs and tested their immunomodulatory effects. As shown in Figure $6 a$ and b, IDO knockdown was effective at both mRNA and protein levels. MSCs with or without IDO knockdown were then co-cultured with human PBMCs in the presence of anti-human CD3 (OKT3). Like mouse MSCs, wild-type human MSCs were capable of promoting anti-CD3-induced proliferation by PBMCs present at low cell densities, whereas suppressing this proliferation at a high cell density (Figure 6c). When IDO knockdown MSCs were used, they were only capable of facilitating anti-CD3stimulated PBMC proliferation, regardless of cell numbers. Therefore, human MSCs act much like mouse MSCs in their ability to modulate immune responses. This provides essential information for better clinical application of human MSCs.

\section{Discussion}

The immunomodulatory properties of MSCs have attracted much attention by both basic and clinical researchers in the past few years. Various mediators have been proposed to be responsible for the immunosuppressive capacity of MSCs, including IL-10, ${ }^{15}$ TGF- $\beta,{ }^{16}$ IDO,${ }^{17}$ TSG $^{18}$ and PG E2. ${ }^{19}$ We recently reported that the concerted action of iNOS and chemokines are critical for MSC-mediated immunosuppression. ${ }^{20}$ Due to their strong immunomodulatory effects, MSCs are being tested in the treatment of immune disorders, such as experimental autoimmune encephalomyelitis (EAE), GvHD and diabetes, in both animal models and humans. Many clinical trials have been initiated according to a recent report. ${ }^{29}$ Importantly, however, the immunosuppressive potential of MSCs is not always achieved. For example, MSCs were found not to suppress GvHD in vivo. ${ }^{21}$

Our previous studies revealed that MSC-mediated immunosuppression is exerted through the coordinated action of chemokines and NO. It is possible that, under conditions that do not promote much NO production, MSC-secreted chemokines might actually enhance immune responses. In the present study, we found that MSCs are immunosuppressive only when exposed to sufficiently high levels of proinflammatory cytokines, as previously described; otherwise they actually promoted lymphocyte proliferation. This result is consistent with a report by another group, showing that MSCs could promote the proliferation of suboptimally activated T cells. ${ }^{30}$ Our study shows that a lack of iNOS activity can switch MSCs from being potently immunosuppressive to being highly immune-enhancing. By employing the mouse DTH model, we confirmed that the absence of iNOS activity prevents MSCs from suppressing the activity of recruited immune cells, and that these recruited cells instead provide a boost to the local immune responses in vivo. Furthermore, this immune-enhancing effect was significantly reduced in $\mathrm{CCR}^{-/-} \mathrm{CXCR}^{-/-}$mice, indicating a crucial role for chemotaxis in $\mathrm{NNOS}^{-1-} \mathrm{MSC}$-mediated immune enhancement. Importantly, iNOS ${ }^{-1-} \mathrm{MSC}^{-}$efficiently prevented tumor growth in a mouse melanoma model, and L-NMMA administration enabled wild-type MSCs to inhibit tumor growth. Furthermore, modulation of IDO expression can also switch human MSCs from being immunosuppressive to be immune-enhancing. Taken together, these results suggest the following scenario: upon stimulation by proinflammatory cytokines, MSCs secrete chemokines that recruit the leukocytes, which could then facilitate the immune response. The presence or absence of NO determines whether or not the recruited leukocytes are inhibited, thereby acting as a switch in MSC-mediated immunomodulation. The dual and opposing roles of MSCs under different circumstances may serve to regulate immune responses generally, a possibility with important implications for current clinical application of MSCs.

During MSC-mediated immunomodulation, proinflammatory cytokines have been shown to have an essential role. ${ }^{14,31}$ In both mice and humans, the co-presence of IFN $\gamma$ and another cytokine, either TNF $\alpha, \mathrm{IL}-1 \alpha$ or IL-1 $\beta$, were required for MSCs to exert their immunosuppressive effect. In all of these experiments, recombinant cytokines or supernatants from activated splenocytes or PBMCs were used to treat MSCs. In their clinical application for the treatment of immune disorders, however, administrated MSCs might encounter insufficient proinflammatory cytokines or a biased cytokine milieu in vivo. When the immunosuppressive potential of MSCs could be achieved would be difficult to predict. NO produced by iNOS is very common and important in the immune system. iNOS activity is reported to be reduced in many cases, including Trichinella spiralis infection, adenovirus E1A protein, and Helicobacter pylori infection. ${ }^{32-34}$ Other factors, like cytokines, some compounds (e.g., betaCarotene and alpha-iso-cubebenol) and glucocorticoid hormones have also been found to inhibit iNOS activity. ${ }^{35-37}$ When applying MSCs in disease models in mice, the immunosuppressive effect might easily be turned off, or even switched to stimulatory effect. Therefore, in some cases, administration of MSCs could fail to ameliorate immune disorders. ${ }^{21,38}$ It may be possible to harness the immuneenhancing effect of MSCs to treat diseases where immune responses have been suppressed. In some studies, iNOS inhibitors were found to be capable of suppressing tumor growth in mice by promoting lymphocyte infiltration. ${ }^{39}$ Interestingly, MSCs have an adverse effect on immunosuppressant inhibition of PBMCs proliferation. ${ }^{24}$ We found that when cyclosporine A was added to the co-culture of MSCs with antiCD3-activated splenocytes, T-cell proliferation was restored at suboptimal concentrations of cyclosporine A (data not shown). Cyclophosphamide, an immunosuppressive drug, has also been reported to be immunostimulatory at low doses, deriving from its inhibition of iNOS. ${ }^{40}$ Herein, our findings provide a reasonable explanation for these effects, as MSCs usually migrate to sites of inflammation, and insufficient levels of proinflammatory cytokines or iNOS activity would render MSCs immune-enhancing. The results in two in vivo models support our hypothesis, as they show that iNOS ${ }^{-1-}$ MSCs can both significantly boost DTH responses and suppress the growth of the melanoma tumors.

Our findings suggest that it is important to be aware of the potential differential effects of cytokines or drugs on the expression and activity of iNOS (or IDO) when applying MSCs in the treatment of disease, as they are a critical switch that determines the immunomodulatory fate of MSCs. 


\section{Materials and Methods}

Mice. C57BL/6, BALB/C and NOD-SCID mice were purchased from the Shanghai Laboratory Animal Center of Chinese Academy of Sciences, Shanghai, China, and maintained under specific pathogen-free conditions. CCR5 ${ }^{-l-}$ mice $\left(C \mathrm{Cr} 5^{\text {tm } 1 k u z}\right)$, $\mathrm{CXCR}^{-1-}$ mice (CXCr3 $\left.3^{\text {tm1Dgen }}\right)$ and iNOS $^{-1-}$ mice (Nos2 ${ }^{\text {tm1 Lau }}$ ) were from Jackson Laboratory (Bar Harbor, ME, USA). Mice were maintained in the Vivarium of Shanghai Jiao Tong University School of Medicine. In our facility, we intercross $\mathrm{CCR}^{-1-}$ mice with $\mathrm{CXCR3^{-1- }}$ mice and then $\mathrm{F} 1$ offspring were bred to each other to generate $C C R 5^{-1-} \mathrm{CXCR}^{-1-}$ mice. Animals were matched for age and gender in each experiment. All procedures were approved by the Institutional Animal Care and Use Committee of the Institute of Health Sciences, Shanghai Institutes for Biological Sciences of Chinese Academy of Sciences.

Reagents. Recombinant mouse IFN $\gamma, \mathrm{TNF} \alpha, \mathrm{IL}-1 \alpha$ and $\mathrm{IL}-1 \beta$ were from eBiosciences (La Jolla, CA, USA). Anti-CD95 (Jo2) was from BD Pharmingen (San Diego, CA, USA). Propidium iodide, Saponin, S-Nitroso-N-acetyl-DL-penicillamine (SNAP), carboxy fluorescein diacetate succinimidyl ester (CFSE), complete Freud's adjuvant and L-NMMA were from Sigma-Aldrich (St. Louis, MO, USA). Human IDO shRNA lentiviral particles and control shRNA lentiviral particles were from Santa Cruz Biotechnology (Santa Cruz, CA, USA). Human IDO and GAPDH monoclonal antibodies were from Cell Signaling Technology (Danvers, MA, USA). Mouse CD3 polyclonal antibody was from Abcam (Cambridge, MA, USA). Diaminobenzidine Histochemistry Kit was from Maixin biotechnology (Fuzhou, Fujian). SMT and 1400w were from Beyotime biotechnology (Shanghai, China).

Cells. MSCs were generated from tibia and femur bone marrow of 6-10-week-old mice. Cells were cultured in DMEM medium supplemented with $10 \% \mathrm{FBS}, 2 \mathrm{mM}$ glutamine, $100 \mathrm{U} / \mathrm{ml}$ penicillin, and $100 \mu \mathrm{g} / \mathrm{ml}$ streptomycin (all from Invitrogen, Carlsbad, CA, USA). Non-adherent cells were removed after $24 \mathrm{~h}$, and adherent cells were maintained with medium replenishment every 3 days. To obtain MSC clones, cells at confluence were harvested and seeded into 96 -well plates by limited dilution. Individual clones were then picked and expanded. Human umbilical cord-derived MSCs were generously provided by Dr. Lingyun Sun, The Affiliated Drum Tower Hospital of Nanjing University Medical School. Cells were used at 5th to 15th passage and all control cells were from similar passages.

T-cell blasts were generated from naïve splenocytes. Cells $\left(1 \times 10^{6} \mathrm{cell} / \mathrm{s} / \mathrm{ml}\right)$ were activated by plastic-bound anti-CD3 and soluble anti-CD28 for $48 \mathrm{~h}$, then cultured with IL-2 $(200 \mathrm{U} / \mathrm{ml})$ alone for $48 \mathrm{~h}$. All T-cell cultures were maintained in RPMI-1640 medium supplemented with $10 \%$ heat-inactivated FBS, $2 \mathrm{mM}$ glutamine, $100 \mathrm{U} / \mathrm{ml}$ penicillin, $100 \mu \mathrm{g} / \mathrm{ml}$ streptomycin, and $50 \mu \mathrm{M} \beta$-ME (complete medium).

Proliferation assay. To assay cell proliferation, $0.5 \mu \mathrm{Ci}$. of ${ }^{3} \mathrm{H}$-thymidine (Tdr, Shanghai Institute of Applied physics, Chinese Academy of Sciences, China) was added to each well $4-6 \mathrm{~h}$ before termination of the cultures by freezing. Plates were then thawed, harvested, and incorporated ${ }^{3} \mathrm{H}-\mathrm{Tdr}$ was assessed using a Wallac Microbeta scintillation counter (Perkin-Elmer, Waltham, MA, USA).

CFSE staining. T-cell blasts $\left(10^{6} / \mathrm{ml}\right.$ in phosphate-buffered saline) were labeled with $5 \mu \mathrm{M}$ CFSE for $8 \mathrm{~min}$ at $37^{\circ} \mathrm{C}$ with gentle vortex every $2 \mathrm{~min}$. Labeling was terminated by adding equal volume of fetal calf serum. After washing, cells were cultured with MSCs. Cell division, as evidenced by reduction of fluorescence intensity by one-half, was analyzed by flow cytometry.

Real-time PCR. Total RNA was isolated using RNAprep pure Cell/Bacteria Kit (Tiangen biotech, Beijing, China), First-strand cDNA synthesis was performed using 1st cDNA Synthesization Kit with oligo(dT) ${ }_{15}$ (Tiangen Biotech). The levels of mRNA of genes of interest were measured by real-time PCR (7900 HT by Applied Biosystems, Foster City, CA, USA) using SYBR Green Master Mix (TaKaRa Biotech, Dalian, China). Total amount of mRNA was normalized to endogenous $\beta$-actin mRNA. Sequences of PCR primer pairs were as follows: mouse CCL2, forward $5^{\prime}$-TCTCTCTTCCTCCACCACCATG- $3^{\prime}$ and reverse $5^{\prime}$-GCGTTAACTGCA TCTGGCTGA-3'; mouse CCL5, forward 5'-TTTCTACACCAGCAGCAAGTGC- $3^{\prime}$ and reverse $5^{\prime}$-CCTTCGTGTGACAAACACGAC- $3^{\prime}$; mouse CXCL9, forward $5^{\prime}$-AG TGTGGAGTTCGAGGAACCCT- $3^{\prime}$ and reverse $5^{\prime}$-TGCAGGAGCATCGTGCAT T-3'; mouse CXCL10, forward $5^{\prime}$-TCCTTGTCCTCCCTAGCTCA- $3^{\prime}$ and reverse $5^{\prime}$-ATAACCCCTTGGGAAGATGG-3'; mouse $\beta$-actin, forward $5^{\prime}$-CCACGAGCGGT
TCCGATG- $3^{\prime}$ and reverse $5^{\prime}$-GCCACAGGATTCCATACCCA-3'; mouse IL-6, forward $5^{\prime}$-AGATAAGCTGGAGTCACAGAAGGAG-3' and reverse $5^{\prime}$-CGCACTAG GTTTGCCGAGTAG-3'; mouse iNOS, forward 5'-CAGCTGGGCTGTACAAAC CTT-3' and reverse $5^{\prime}$ - CATTGGAAGTGAAGCGTTTCG-3'; mouse eNOS, forward $5^{\prime}$-TCAGCCATCACAGTGTTCCC-3' and reverse $5^{\prime}$-ATAGCCCGCATAGCGTATCA G-3'; mouse nNOS, forward $5^{\prime}$-CCCAACGTCATTTCTGTCCGT-3' and reverse 5'-CTGACCCGTTCCTTCACC-3'; human IDO, forward 5'-TGCCCCTGTGATAAA CTGTGGT-3' and reverse $5^{\prime}$-CATTCTTGTAGTCTGCTCCTCTGG-3'; human iNOS, forward $5^{\prime}$-CCTGAGCTCTTCGAAATCCCA-3' and reverse $5^{\prime}$-CCCGAAAC CACTCGTATTTGG-3'; human eNOS, forward $5^{\prime}$-TGATGGCGAAGCGAGTGAA G-3' and reverse $5^{\prime}$-CTGCTGTGCGTAGCTCTGG-3'; human nNOS, forward $5^{\prime}$ GAAGGAGCGGGTCAGTAAGC-3' and reverse $5^{\prime}$-CCCCACGAATCAGGTCAGAG A-3'; human CXCL9, forward 5'-GGTTCTGATTGGAGTGCAAGGA-3' and reverse 5'-GGATAGTCCCTTGGTTGGTGCT-3'; human CXCL10, forward 5'-GCCAATTTT GTCCACGTGTTG-3' and reverse $5^{\prime}$-GGCCTTCGATTCTGGATTCAG-3'; human CXCL11, forward 5'-CCTTGGCTGTGATATTGTGTGC-3' and reverse $5^{\prime}$-CCTATG CAAAGACAGCGTCCT-3'.

DTH response. C57BL/6 mice (6-10 weeks old) were immunized by tail-base injection of OVA(10 $\mu \mathrm{g}$ in $50 \mu \mathrm{l}$ saline) emulsified with $50 \mu \mathrm{l}$ complete Freund's adjuvant. DTH was tested after 6 days, by challenging with 200 or $20 \mu \mathrm{g}$ aggregated OVA in $30 \mu \mathrm{l}$ saline injected into the right hind footpad. The left footpad was injected with $30 \mu \mathrm{l}$ of saline with or without MSCs as a negative control. After $24 \mathrm{~h}$, antigeninduced footpad thickness increment was measured using a caliper (no. 7308, Mitutoyo, Tokyo, Japan) and calculated as: thickness increment $=R-L$, where $R$ and $L$ are thickness of right and left footpads, respectively.

Mouse melanoma model. B16-F0 mouse melanoma cells were expanded in complete DMEM medium in vitro. Each mouse was injected with $0.5 \times 10^{6} \mathrm{~B} 16$-F0 in $100 \mu$ l PBS intramuscularly on the left thigh, with or without co-injection of wildtype MSCs or iNOS ${ }^{-1-}$ MSCs $\left(1 \times 10^{6}\right.$ cells). MSCs were again administrated at day 3,6 and 9 at the same sites, with PBS serving as a control. Mice were observed daily and euthanized when tumor began to significantly affect mobility. The melanoma tumors were then excised and weighed. $4 \mu \mathrm{m}$ paraffin sections were prepared and stained with indicated antibody according to the protocol provided by the manufacturer.

Western blot. Protein samples in SDS sample buffer were heated at $95^{\circ} \mathrm{C}$ for $10 \mathrm{~min}$ and separated on SDS-polyacrylamide gel. Then proteins were electroblotted to nitrocellulose transfer membranes and revealed by mouse and rabbit antibodies against IDO or GAPDH overnight at $4{ }^{\circ} \mathrm{C}$. Finally, the blot was subjected to chemiluminescent detection according to the manufacturer's instructions.

Statistical analysis. Data are presented as mean \pm S.D. Statistical significance was assessed by unpaired two-tailed Student's $t$-test.

\section{Conflict of Interest}

The authors declare no conflict of interest.

Acknowledgements. We thank Dr. Lingyun Sun for the human umbilical cord mesenchymal stem cells. This work was supported by Ministry of Science and Technology of China (2010CB945600, 2011DFA30630 and 2009ZX09503-024), Scientific Innovation Project of the Chinese Academy of Science (XDA01040000 and KSCX1-YW-22), the National Science Foundation of China (31010103908), Shanghai Pujiang Program of China (PJ (2009) 01267) and the National Institutes of Health (DE014913, DE019932 and GM866889).

\section{Author contributions}

WL, GR: conception and design, collection and assembly of data, data analysis and interpretation, manuscript writing; LZ, ZR, YH, JS, YH: collection and assembly of data, data analysis and interpretation; JL, XC, KC, QC, PS: data analysis and interpretation; AIR: manuscript writing; SS, AL: conception and design, data analysis and interpretation; YS: conception and design, manuscript writing, final approval of manuscript, financial support. 
1. Macmillan ML, Blazar BR, DeFor TE, Wagner JE. Transplantation of ex-vivo culture expanded parental haploidentical mesenchymal stem cells to promote engraftment in pediatric recipients of unrelated donor umbilical cord blood: results of a phase I-II clinical trial. Bone Marrow Transplant 2009; 43: 447-454.

2. Le Blanc K, Frassoni F, Ball L, Locatelli F, Roelofs H, Lewis I et al. Mesenchymal stem cells for treatment of steroid-resistant, severe, acute graft-versus-host disease: a phase II study. Lancet 2008; 371: 1579-1586.

3. Le Blanc K, Rasmusson I, Sundberg B, Gotherstrom C, Hassan M, Uzunel M et al. Treatment of severe acute graft-versus-host disease with third party haploidentical mesenchymal stem cells. Lancet 2004; 363: 1439-1441.

4. Vojtassak J, Danisovic L, Kubes M, Bakos D, Jarabek L, Ulicna M et al. Autologous biograft and mesenchymal stem cells in treatment of the diabetic foot. Neuro Endocrinol Lett 2006; 27 (Suppl 2): 134-137.

5. Christopeit M, Schendel M, Foll J, Muller LP, Keysser G, Behre G. Marked improvement of severe progressive systemic sclerosis after transplantation of mesenchymal stem cells from an allogeneic haploidentical-related donor mediated by ligation of CD137 L. Leukemia 2008; 22: 1062-1064.

6. Guiducci S, Porta F, Saccardi R, Guidi S, Ibba-Manneschi L, Manetti M et al. Autologous mesenchymal stem cells foster revascularization of ischemic limbs in systemic sclerosis: a case report. Ann Intern Med 2010; 153: 650-654.

7. Kharaziha P, Hellstrom PM, Noorinayer B, Farzaneh F, Aghajani K, Jafari F et al. Improvement of liver function in liver cirrhosis patients after autologous mesenchymal stem cell injection: a phase I-II clinical trial. Eur J Gastroenterol Hepatol 2009; 21: 1199-1205.

8. Sun L, Wang $\mathrm{D}$, Liang J, Zhang $\mathrm{H}$, Feng $\mathrm{X}$, Wang $\mathrm{H}$ et al. Umbilical cord mesenchymal stem cell transplantation in severe and refractory systemic lupus erythematosus. Arthritis Rheum 2010; 62: 2467-2475.

9. Duijvestein M, Vos AC, Roelofs H, Wildenberg ME, Wendrich BB, Verspaget HW et al. Autologous bone marrow-derived mesenchymal stromal cell treatment for refractory luminal Crohn's disease: results of a phase I study. Gut 2010; 59: 1662-1669.

10. Garcia-Olmo D, Garcia-Arranz M, Herreros D, Pascual I, Peiro C, Rodriguez-Montes JA. A phase I clinical trial of the treatment of Crohn's fistula by adipose mesenchymal stem cell transplantation. Dis Colon Rectum 2005; 48: 1416-1423.

11. Rasmusson I, Ringden O, Sundberg B, Le Blanc K. Mesenchymal stem cells inhibit lymphocyte proliferation by mitogens and alloantigens by different mechanisms. Exp Cell Res 2005; 305: 33-41.

12. Sato K, Ozaki K, Oh I, Meguro A, Hatanaka K, Nagai T et al. Nitric oxide plays a critical role in suppression of T-cell proliferation by mesenchymal stem cells. Blood 2007; 109: 228-234.

13. Xu G, Zhang L, Ren G, Yuan Z, Zhang Y, Zhao RC et al. Immunosuppressive properties of cloned bone marrow mesenchymal stem cells. Cell Res 2007; 17: 240-248.

14. Ren G, Zhang L, Zhao X, Xu G, Zhang Y, Roberts Al et al. Mesenchymal stem cell-mediated immunosuppression occurs via concerted action of chemokines and nitric oxide. Cell Stem Cell 2008; 2: 141-150.

15. Batten P, Sarathchandra P, Antoniw JW, Tay SS, Lowdell MW, Taylor PM et al. Human mesenchymal stem cells induce T cell anergy and downregulate $T$ cell allo-responses via the $\mathrm{TH} 2$ pathway: relevance to tissue engineering human heart valves. Tissue Eng 2006 12: 2263-2273.

16. Groh ME, Maitra B, Szekely E, Koc ON. Human mesenchymal stem cells require monocytemediated activation to suppress alloreactive T cells. Exp Hematol 2005; 33: 928-934.

17. Meisel R, Zibert A, Laryea M, Gobel U, Daubener W, Dilloo D. Human bone marrow stromal cells inhibit allogeneic T-cell responses by indoleamine 2,3-dioxygenase-mediated tryptophan degradation. Blood 2004; 103: 4619-4621.

18. Lee RH, Pulin AA, Seo MJ, Kota DJ, Ylostalo J, Larson BL et al. Intravenous hMSC improve myocardial infarction in mice because cells embolized in lung are activated to secrete the anti-inflammatory protein TSG-6. Cell Stem Cell 2009; 5: 54-63.

19. Aggarwal S, Pittenger MF. Human mesenchymal stem cells modulate allogeneic immune cell responses. Blood 2005; 105: 1815-1822.

20. Ren G, Su J, Zhang L, Zhao X, Ling W, L'Huillie A et al. Species variation in the mechanisms of mesenchymal stem cell-mediated immunosuppression. Stem Cells 2009; 27: 1954-1962.

21. Sudres M, Norol F, Trenado A, Gregoire S, Charlotte F, Levacher B et al. Bone marrow mesenchymal stem cells suppress lymphocyte proliferation in vitro but fail to prevent graft-versus-host disease in mice. J Immunol 2006; 176: 7761-7767.
22. Nauta AJ, Westerhuis G, Kruisselbrink AB, Lurvink EG, Willemze R, Fibbe WE. Donor-derived mesenchymal stem cells are immunogenic in an allogeneic host and stimulate donor graft rejection in a nonmyeloablative setting. Blood 2006; 108: 2114-2120.

23. Inoue S, Popp FC, Koehl GE, Piso P, Schlitt HJ, Geissler EK et al. Immunomodulatory effects of mesenchymal stem cells in a rat organ transplant model. Transplantation 2006; 81: 1589-1595.

24. Hoogduijn MJ, Crop MJ, Korevaar SS, Peeters AM, Eijken M, Maat LP et al. Susceptibility of human mesenchymal stem cells to tacrolimus, mycophenolic acid, and rapamycin. Transplantation 2008; 86: 1283-1291.

25. Ringden $\mathrm{O}$, Uzunel M, Rasmusson I, Remberger M, Sundberg B, Lonnies $\mathrm{H}$ et al. Mesenchymal stem cells for treatment of therapy-resistant graft-versus-host disease. Transplantation 2006; 81: 1390-1397.

26. Miura Y, Kami M, Tsubokura M, Takei N, Komatsu T. Mesenchymal stem cells for acute graft-versus-host disease. Lancet 2008; 372: 715-716; author reply 716 .

27. Soleimani M, Nadri S. A protocol for isolation and culture of mesenchymal stem cells from mouse bone marrow. Nat Protoc 2009; 4: 102-106.

28. Xu G, Zhang Y, Zhang L, Ren G, Shi Y. The role of IL-6 in inhibition of lymphocyte apoptosis by mesenchymal stem cells. Biochem Biophys Res Commun 2007; 361: 745-750.

29. Trounson A. New perspectives in human stem cell therapeutic research. BMC Med 2009; 7: 29.

30. Renner $\mathrm{P}$, Eggenhofer E, Rosenauer A, Popp FC, Steinmann JF, Slowik $\mathrm{P}$ et al. Mesenchymal stem cells require a sufficient, ongoing immune response to exert their immunosuppressive function. Transplant Proc 2009; 41: 2607-2611.

31. Krampera M, Cosmi L, Angeli R, Pasini A, Liotta F, Andreini A et al. Role for interferongamma in the immunomodulatory activity of human bone marrow mesenchymal stem cells. Stem Cells 2006; 24: 386-398.

32. Bian K, Zhong M, Harari Y, Lai M, Weisbrodt N, Murad F. Helminth regulation of host IL4Ralpha/Stat6 signaling: mechanism underlying NOS-2 inhibition by Trichinella spiralis. Proc Natl Acad Sci USA 2005; 102: 3936-3941.

33. Cao W, Bao C, Lowenstein CJ. Inducible nitric oxide synthase expression inhibition by adenovirus E1A. Proc Natl Acad Sci USA 2003; 100: 7773-7778.

34. Gobert AP, McGee DJ, Akhtar M, Mendz GL, Newton JC, Cheng Y et al. Helicobacter pylori arginase inhibits nitric oxide production by eukaryotic cells: a strategy for bacterial survival. Proc Natl Acad Sci USA 2001; 98: 13844-13849.

35. Lee YJ, Park SY, Kim SG, Park da J, Kang JS, Lee SJ et al. Identification of a novel compound that inhibits iNOS and COX-2 expression in LPS-stimulated macrophages from Schisandra chinensis. Biochem Biophys Res Commun 2010; 391 1687-1692.

36. Kling KM, Kirby L, Kwan KY, Kim F, McFadden DW. Interleukin-10 inhibits inducible nitric oxide synthase in an animal model of necrotizing enterocolitis. Int J Surg Investig 1999; 1 : 337-342.

37. Ozaki T, Habara K, Matsui K, Kaibori M, Kwon AH, Ito S et al. Dexamethasone inhibits the induction of iNOS gene expression through destabilization of its mRNA in proinflammatory cytokine-stimulated hepatocytes. Shock 2010; 33: 64-69.

38. Djouad F, Fritz V, Apparailly F, Louis-Plence P, Bony C, Sany J et al. Reversal of the immunosuppressive properties of mesenchymal stem cells by tumor necrosis factor alpha in collagen-induced arthritis. Arthritis Rheum 2005; 52: 1595-1603.

39. Badn W, Hegardt P, Fellert MA, Darabi A, Esbjornsson M, Smith KE et al. Inhibition of inducible nitric oxide synthase enhances anti-tumour immune responses in rats immunized with IFN-gamma-secreting glioma cells. Scand J Immunol 2007; 65: 289-297.

40. Loeffler M, Kruger JA, Reisfeld RA. Immunostimulatory effects of low-dose cyclophosphamide are controlled by inducible nitric oxide synthase. Cancer Res 2005; 65: $5027-5030$.

This work is licensed under the Creative Commons Attribution-NonCommercial-No Derivative Works 3.0 Unported License. To view a copy of this license, visit http:// creativecommons.org/licenses/by-nc-nd/3.0

\section{Supplementary Information accompanies the paper on Cell Death and Differentiation website (http://www.nature.com/cdd)}

